\title{
The state of robotic cardiac surgery in Europe
}

\author{
Matteo Pettinari ${ }^{1}$, Emiliano Navarra $^{2}$, Philippe Noirhomme ${ }^{2}$, Herbert Gutermann ${ }^{1}$ \\ ${ }^{1}$ Division of Cardiac Surgery, Ziekenhuis Oost Limburg, Genk, Belgium; ${ }^{2}$ Division of Cardiothoracic and Vascular Surgery, St-Luc University \\ Hospital, Catholic University of Louvain, Brussels, Belgium \\ Correspondence to: Matteo Pettinari. Division Cardiac Surgery, Ziekenhuis Oost Limburg, Schiepse Bos 6, 3600, Genk, Belgium. \\ Email: matteo.pettinari@zol.be.
}

\begin{abstract}
Background: In the past two decades, the introduction of robotic technology has facilitated minimally invasive cardiac surgery, allowing surgeons to operate endoscopically rather than through a median sternotomy. This approach has facilitated procedures for several structural heart conditions, including mitral valve repair, atrial septal defect closure and multivessel minimally invasive coronary artery bypass grafting. In this rapidly evolving field, we review the status of robotic cardiac surgery in Europe with a focus on mitral valve surgery and coronary revascularization.

Methods: Structured searches of MEDLINE, Embase, and Cochrane databases were performed from their dates of inception to June 2016. All original studies, except case-reports, were included in this qualitative review. Studies performed in Europe were presented quantitatively. Data provided from Intuitive Surgical Inc. are also presented.

Results: Fourteen papers on coronary surgery were included in the analysis and reported a mortality rate ranging between $0-1 \%$, revision for bleeding between $2-7 \%$, conversion to a larger incision between $2-15 \%$, and patency rate between $92-98 \%$. The number of procedures ranged between 23 and 170 per year. There were only a small number of published reports for robotic mitral valve surgery from European centers.

Conclusions: Coronary robotic surgery in Europe has been performed safely and effectively with very few perioperative complications in the last 15 years. On the other hand, mitral surgery has been developed later with increasing applications of this technology only in the last 5-6 years.
\end{abstract}

Keywords: Minimally invasive; mitral valve; coronary; robotic

Submitted Aug 12, 2016. Accepted for publication Oct 25, 2016.

doi: $10.21037 /$ acs.2017.01.02

View this article at: http://dx.doi.org/10.21037/acs.2017.01.02

\section{Introduction}

In recent decades, the benefits of minimizing surgical trauma, such as reduced pain, shorter hospital stays, faster return to normal activities, and improved cosmesis, have substantially increased interest in minimally invasive surgery. At the same time, improvements in surgical instrumentation, perfusion technology and visioning platforms have facilitated advances in minimally invasive approaches. As a result, minimally invasive procedures have become the standard of care at certain institutions worldwide. However, one disadvantage is that endoscopic instrumentation provides only four degrees of freedom, hence significantly reducing dexterity, which is essential for delicate cardiac procedures. Furthermore, the loss of depth perception from two-dimensional video monitors increases operative difficulty.

In order to overcome the limitations of long-shafted endoscopic instruments, extensive work was undertaken on the development of robotic telemanipulators. Telemanipulators provide three-dimensional (3D) vision and articulating instruments with seven degrees of freedom of motion, similar to the human hand.

Carpentier and Mohr independently performed the first successful robotic mitral valve cases in 1998 using prototypes of the da Vinci Surgical System (Intuitive Surgical Inc., Sunnyvale, California, USA) telemanipulator $(1,2)$. Since then, telemanipulators have continued to evolve. 
The first generation of the da Vinci Surgical System was FDA approved in 1999, and three new generations were subsequently released: the da Vinci S (FDA approved in 2005), Si (FDA approved in 2009) and most recently the $\mathrm{Xi}$ version (FDA approved in 2014). The latest systems provide high resolution $3 \mathrm{D}$ visualization, up to ten-times magnification of the operating field, movement scaling, and dual console systems for surgeon cooperation and training (3).

Currently, over 1,700 robotic cardiac operations are performed in the USA per year with a nearly constant number of procedures in the last 8 years. The purpose of this article is to provide an overview of the experience in robotic cardiac surgery in Europe with a focus on mitral valve surgery and coronary revascularization.

\section{Methods}

\section{Literature review}

Electronic searches were performed on MEDLINE, EMBASE, Cochrane Central Register of Controlled Trials (CCTR), Cochrane Database of Systematic Reviews (CDSR), ACP Journal Club and Database of Abstracts of Review of Effectiveness (DARE) from their dates of inception to June 2016. The search strategy included a combination of 'robotic' or 'telemanipulator' or 'computerassisted' as well as 'mitral' and 'coronary' as keywords. Studies that reported clinical outcomes or learning curve analysis of robotic surgery including mitral valve repair and replacement and coronary surgery were selected for qualitative analysis. When institutions published duplicate trials, only the most updated reports were included for qualitative appraisal. All publications were limited to human subjects, the English language and European centers and authors.

\section{Industry data}

Intuitive Surgical Inc. provided the authors with numerical data on robot-assisted procedures performed in Europe since 1998. The data set were anonymous with regards to patient identity and the surgeon/center performing the operation.

\section{Results}

\section{Mitral valve surgery}

Search methods identified six relevant papers in the mitral surgery group. One study was excluded as it reviewed nonEuropean results, and another because results were included in later studies with cumulative patients. This resulted in four studies for quantitative appraisal.

Carpentier performed the first robotic mitral valve repair (MVr) using an early prototype of the da Vinci surgical system in May 1998 (4); four patients were treated with a ring annuloplasty and in one case, an ASD closure was also performed. All patients survived, with a median hospitalization time of 10 days.

Using the da Vinci system, 23 patients with nonischemic valve insufficiency were successfully repaired $(5,6)$ by the group from Leipzig. The mean age of the patients was $58 \pm 9$ years, and they were treated for anterior and posterior leaflet prolapse. The CPB time was $166 \pm 30 \mathrm{~min}$, and clamping time was $93 \pm 17 \mathrm{~min}$. Two patients were reoperated early; one patient underwent an intraoperative mitral valve replacement after failed repair and a second patient had a mitral valve replacement at day 3 due to annular rupture.

Folliguet (7) treated 25 patients with the da Vinci system between February 2004 and September 2005 and matched them retrospectively with 25 patients who underwent the same repair via a median sternotomy. All patients had successful valve repairs. There were no deaths. Patients in the minimally invasive group had longer cardiopulmonary bypass (122.1 vs. $85.7 \mathrm{~min}, \mathrm{P}=0.003$ ) and clamping time (96.1 vs. 69.6, $\mathrm{P}=0.003)$. The length of stay was less for the minimally invasive group ( $7 v s .9$ days). At postoperative echocardiography, two patients in both groups had developed 2+ mitral regurgitations. All other patients had a competent mitral valve repair with no insufficiency. All patients were in NYHA Class I and remained so at 12 and 24 months' follow-up.

Of the 102 robotic cardiac procedure at St. Mary's Hospital in London, only one patient was treated with mitral valve repair (8). The mean length of stay for the 102 patients was 3.1 days and no mortality was recorded.

Recently Musumeci (9) presented a periareolar technique used in five patients: no hospital mortality was registered and no postoperative complications were observed. All patients were discharged before postoperative day 5 .

Following the early trials of the da Vinci system, the number of robot-assisted mitral valve repairs decreased, with a nearly complete abandon of this approach in Europe until 2010 (between 1998 and 2010 the cumulative number of mitral valve procedures performed was 164). However, since 2010, this number has increased exponentially with 
Mitral valve procedures

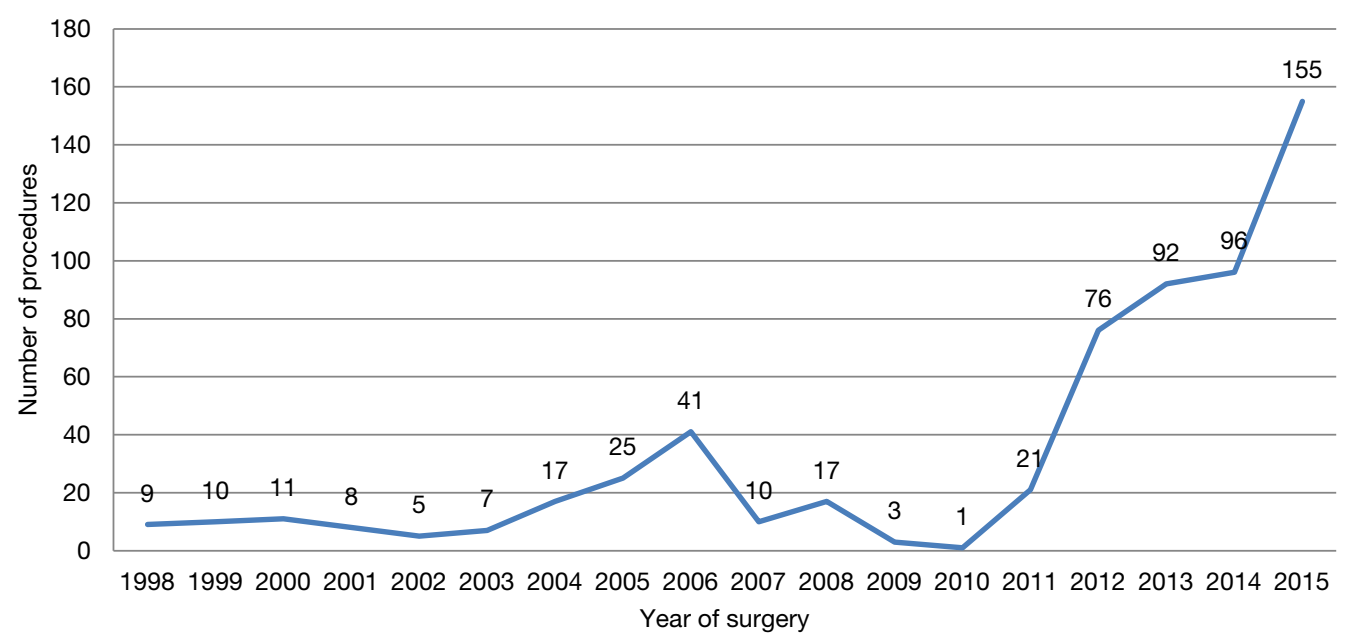

Figure 1 Mitral valve procedures in Europe.

440 procedures performed between January 2011 and December 2015; 70 procedures have been performed in the first 6 months of 2016 (Figure 1) (data provided by Intuitive Surgical Inc). Since 1998, 14 centers have performed robotic-assisted mitral valve surgery in six countries: Finland, the Netherlands, Belgium, France, Principality of Monaco and Italy.

\section{Coronary surgery}

Following a literature search, 64 papers met the inclusion criteria. After exclusion of manuscripts with overlapping patients populations, and studies with less than three patients, 14 papers were selected.

Loulmet (10) performed the first-in-man robotic total endoscopic coronary bypass (TECAB) in Paris using the first-generation da Vinci robotic system on four men in 1998. The Heartport system (Heartport, Redwood City, CA, USA) was used to arrest the heart during anastomosis, and the entire operation was completed endoscopically with robotic-assisted instruments. Early postoperative coronary angiography demonstrated the patency of the grafts in all cases. All patients were symptom-free at 6-month follow-up.

One of the first multi-vessel robotic-assisted bypass series using the da Vinci Surgical System was reported by Cichon (11) in Dresden, Germany in 2000. This team treated 17 (four women, 13 men; median age $63 \pm 7.4$ years) patients with multi-vessel coronary artery disease using bilateral internal thoracic artery (BITA). A 100\% survival rate was documented, and operative time was $255 \pm 40.4$ minutes. BITA harvesting took $88.5 \pm 15.9 \mathrm{~min}$ and cross clamp time was $36 \pm 8.7 \mathrm{~min}$. An average of 2.06 anastomoses were performed per patient. One patient $(5.8 \%)$ required reexploration for bleeding.

Over subsequent years, several European centers began to adopt the robotic approach. In 2001, Mohr (12) from Leipzig, Germany, published a large series of 131 patients who underwent various robotic-assisted CABG; the system was used in three ways; (I) to take down the left internal thoracic artery (ITA) followed by a minimally invasive direct coronary artery bypass (MIDCAB-off-pump handsewn anastomosis of the LITA to the LAD via a minithoracotomy) ( $\mathrm{n}=81$ ); (II) anastomosis between the ITA and the LAD utilizing sternotomy and CPB $(n=15)$; or (III) for TECAB grafting where the LITA was robotically anastomosed to the LAD on the arrested $(\mathrm{n}=27)$ or beating heart $(n=8)$. The ITA was successfully harvested in $97.5 \%$ of the patients in the group undergoing CABG. After the initial period of learning, it was performed in less than $40 \mathrm{~min}$. The postoperative patency rate was $96.3 \%$. TECAB was completed in 22 of 27 cases, with $95.4 \%$ patency demonstrated by angiography at 3-month follow-up.

In 2001, Kappert (13) reported use of the Da Vinci system in 201 patients (156 men and 45 women, median age $64 \pm 10.5$ years, left ventricular ejection fraction $68 \pm 12.4 \%$ ). In this study, patients were divided into three groups. Group A ( $n=156)$ consisted of patients for which the robotic system was used to harvest the left or right internal 
mammary artery, or both. The anastomoses were performed directly through a small chest incision. In group B $(n=37)$, harvest of the internal mammary arteries and the coronary anastomoses were performed totally endoscopically. In group $C$, patients $(n=8)$ were treated with robotic-enhanced CABG via a median sternotomy already preoperatively planned. The survival rate was $99.4 \%$. One patient $(0.6 \%)$ died due to pneumonia on postoperative day 16. The conversion rate to median sternotomy was $5 \%$. The left and right internal mammary artery conduits were successfully harvested in $98 \%$ and $100 \%$, respectively. Increasing experience significantly reduced the time of dissection of the left internal mammary artery alone. Nine patients (4.5\%) were reported to have bleeding requiring re-exploration. All patients were discharged from the hospital after a mean of 7 days.

Four years following the publication of Kappert's findings, Bonatti (14) reported a series of 107 cases of robotic endoscopic CABG in patients with single- and multi-vessel CAD. Robotically assisted LITA harvesting was performed in all patients with completion of the procedure as conventional CABG, MIDCAB, or OPCAB $(\mathrm{n}=22)$, robotically assisted suturing of LITA to LAD during conventional CABG $(\mathrm{n}=28)$, TECAB on the arrested heart using remote-access perfusion $(n=48)$, TECAB on the beating heart using an endo stabilizer $(\mathrm{n}=8)$, and a takedown of adhesions (TECAB intended) $(n=1)$. There was no hospital mortality. Undesirable surgical events (USE) such as conversion to sternotomy or postoperative revision of the anastomosis occurred in 34 of 107 (32\%) patients. The median ventilation time and ICU stay were 11 [0-278] and 21 [11-389] hours, respectively. Cumulative 3-year survival was $100 \%$ and freedom from angina at 3 years was $97 \%$.

Following on from the aforementioned study, the results from a multicenter European study were published in 2007 (15). Between September 1998 and November 2002, 228 patients with CAD underwent TECAB using da Vinci Surgical System (Intuitive Surgical) at five European institutions. Patients underwent TECAB with either an onpump (group $A, n=117$ ) or an off-pump approach (group $B$, $\mathrm{n}=111$ ) and were followed for 6 months. Procedural feasibility was demonstrated via 164 successful endoscopic cases; 64 patients (group C, 28\%) had conversion to nonrobotic procedures. The overall procedural efficacy, as defined by angiographic patency or lack of ischemic signs on stress electrocardiography, was $97 \%$. The incidence of major adverse cardiac events within 6 months was $5 \%$.

The Bonatti group in Innsbruck reported the largest
European series to date in 2011 (16). This group performed robotic TECAB in 326 patients (age, 60 years; range, 31 to 90 years); 242 with single-vessel and 84 with multi-vessel TECAB. Forty-six of 326 patients $(14 \%)$ were converted to a larger incision (minithoracotomy, $\mathrm{n}=5$; sternotomy, $\mathrm{n}=41$ ). Adverse outcomes such as hospital mortality was found to be $0.6 \%$, postoperative stroke rate was $2 \%$, renal failure $0.6 \%$, and perioperative myocardial infarction was $2.5 \%$. Long-term freedom from MACCE was $84 \%, 84 \%$, and $81 \%$ at 1 year, 3 , and 5 years, respectively. A summary of the results of robot-assisted coronary surgery in Europe is presented in Table 1 .

Since the Da Vinci system became available, the annual number of robot-assisted CABG in Europe has been stable; between 2000 and 2010, 1,262 procedures were performed (Figure 2). After 2011, there was a decline in number of procedures performed; however, this was followed by a subsequent increase in annual procedures over the last 3 years (Figure 2).

\section{Discussion}

The evolution of robotic cardiac surgery (both in mitral and coronary surgery) stemmed from a small number of European centers. After an initial spread, the use of the Da Vinci in mitral valve surgery was virtually abandoned for more than a decade. However, the number of robot-assisted coronary procedures remained stable since its introduction. A possible explanation for this period of quiescence in robotic mitral surgery is the contemporary development of the Port-access system in the late 1990s (17). This technology came into widespread use in many European centers (18-20). After an initial phase of learning and development, surgeons from multiple centers demonstrated that this technique could be performed with low perioperative complication rates and acceptable durability $(21,22)$. The Port-access technique was introduced over Europe with ease, perhaps due to the low initial costs compared with the da Vinci system. A significant barrier to the introduction of the da Vinci system has been the purchase price, which may exceed $\$ 1.5$ million, as well as an annual service contract that ranges between $\$ 100,000$ to $\$ 140,000$ (23).

When considering the initial capital investment for the robotic surgical system through amortization of institutional costs, the cost was significantly higher for robotic operations. However, while the absolute cost for robotic surgery was higher than conventional techniques, 


\begin{tabular}{|c|c|c|c|c|c|c|c|c|c|}
\hline Cichon & 17 & MIDCAB & 0 & 0 & 6 & & 0 & - & - \\
\hline Mohr & 131 & 15 sternotomy; 81 MIDCAB; 35 TECAB & 2 & 0 & 2 & 1 & 2 & 9.4 & 95.40 \\
\hline Torracca & 12 & MIDCAB & 8.3 & 0 & 8.3 & 0 & 0 & 7 & - \\
\hline Bonatti & 107 & 22 MIDCAB; 28 sternotomy; 57 TECAB & - & 0 & - & - & - & - & - \\
\hline Loisance & 60 & Mostly sternotomy; 13 TECAB & 15 & 2 & - & - & - & - & - \\
\hline Nesher & 146 & MIDCAB & - & 0 & - & 0 & 0 & 8 & - \\
\hline Folliguet & 56 & 24 MIDCAB; 23 TECAB & 16 & 2 & 4 & - & - & 7.1 & - \\
\hline Schachner & 326 & TECAB & 13 & 0,6 & - & 2 & 2 & 6 & - \\
\hline Sabashnikov & 236 & 189 MIDCAB; 76 ACAB; 236 robot assisted CABG & 3.8 & 1.3 & 3 & - & 1 & - & - \\
\hline
\end{tabular}

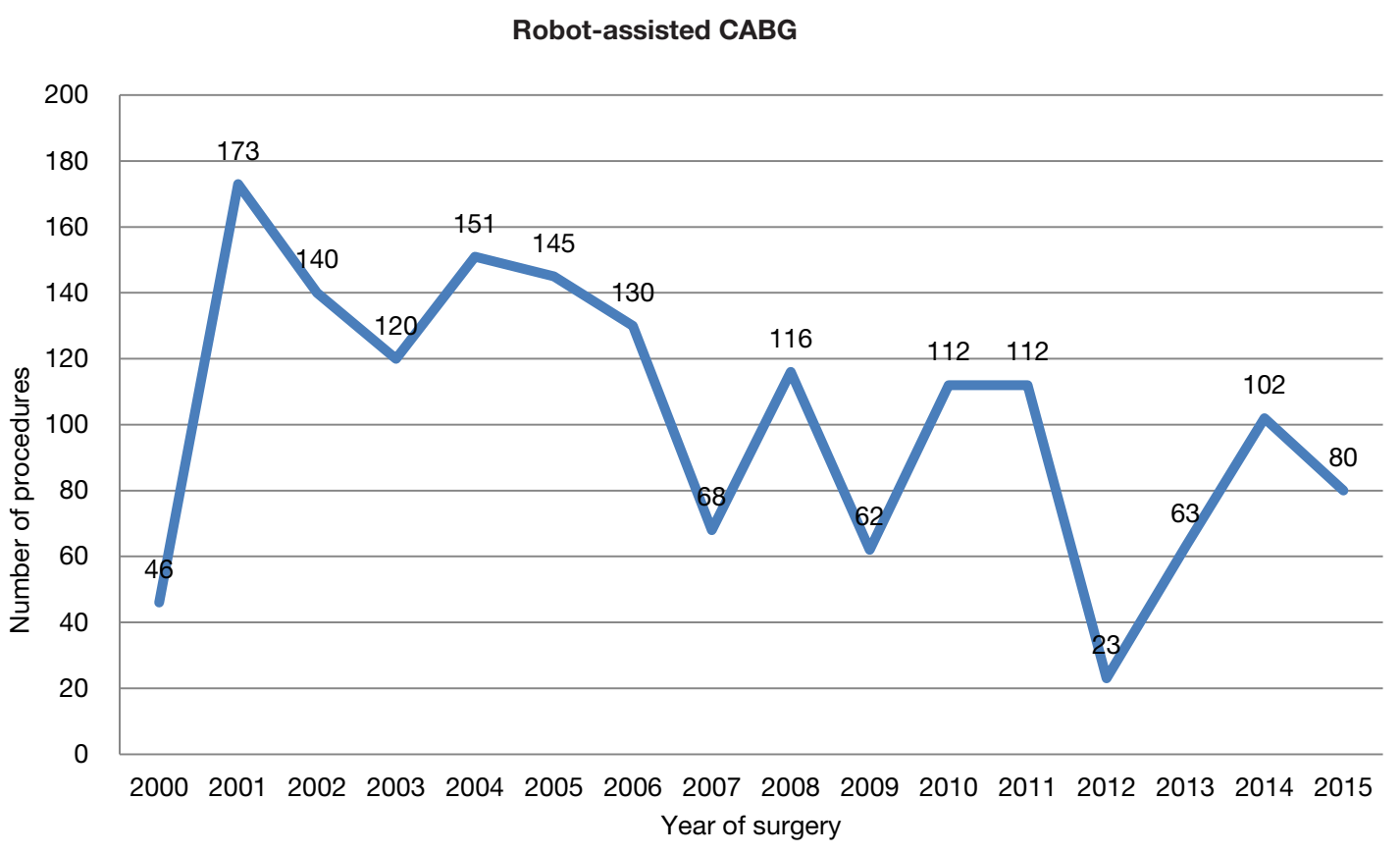

Figure 2 Robot-assisted CABG in Europe. CABG, coronary artery bypass grafting. 


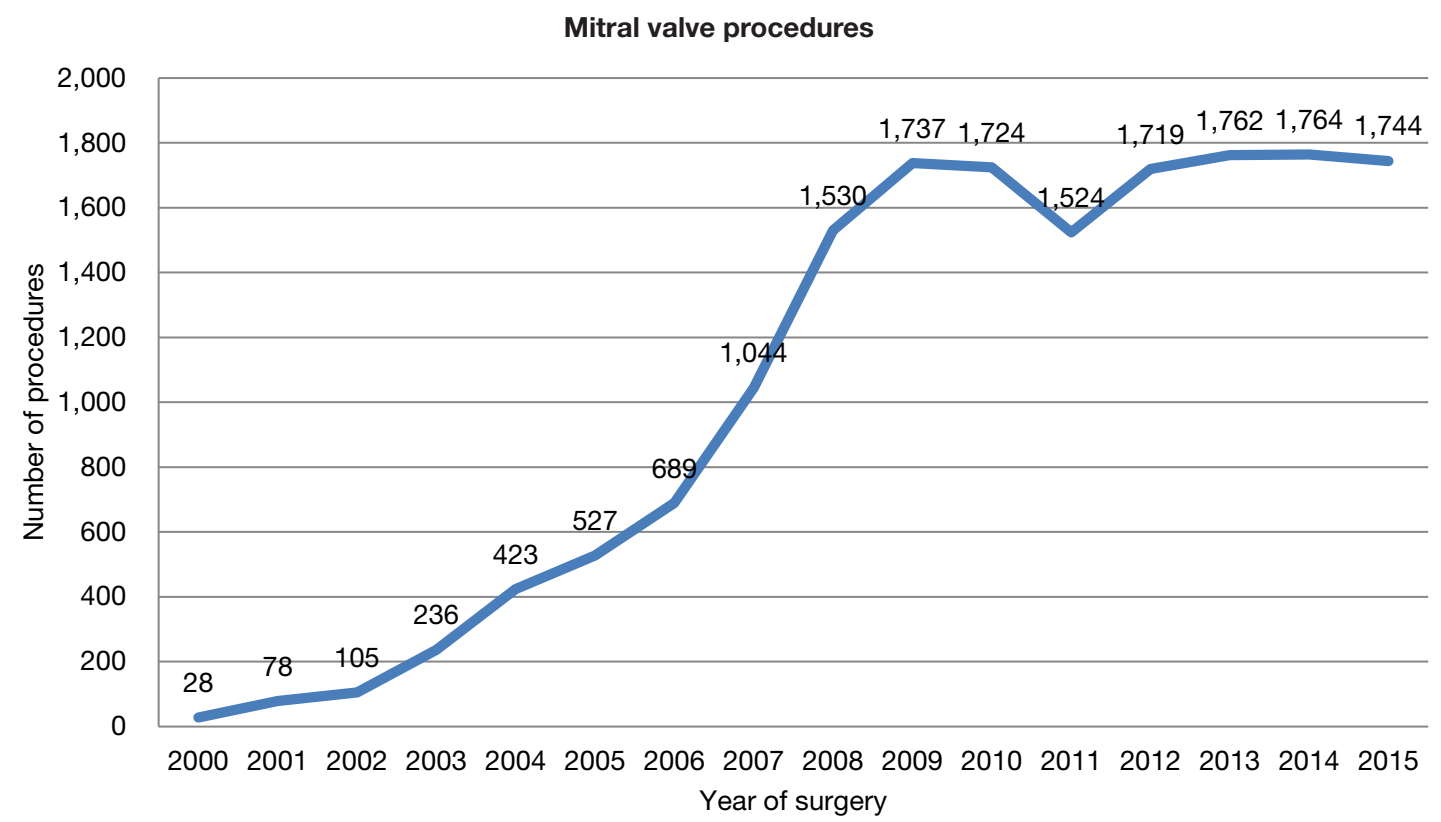

Figure 3 Mitral valve procedures in the United States.

the major driver of cost (OR time) decreased over time, resulting in a significant reduction in the associated cost of this technology. This was highlighted by Morgen (24), who demonstrated that robotic technology did not significantly increase operational total hospital costs.

The lack of tactile feedback during suturing was initially considered to be an important limiting factor in robotic surgery. However, the enhanced 3D visual feedback from the Da Vinci system can achieve similar outcomes through the observation of tissue displacement and deformation (25). Moreover, the system greatly improves operative visualization via $3 \mathrm{D}$ high definition imaging. Visualization of the mitral valve and subvalvular system in particular is unparalleled when using the da Vinci system versus endoscopic minimally invasive approaches. The da Vinci System also provides increased operative dexterity for surgeons. Articulating instruments move with seven degrees of freedom, as compared with the four degrees of freedom provided by endoscopic instruments. The robotic instruments permit tremor-free movements, ambidexterity, and avoidance of the fulcrum effect that is inherent when using long-shafted endoscopic instruments.

The penetration of robotic technology in North America was initially limited to a small number of highly specialized, high-volume centers that developed a standardized technique. More recently, a large number of experienced groups have collectively reported on several thousand patients who have undergone robot-assisted MV repair with low hospital mortality rates as well as low rates of complications including stroke, re-exploration for bleeding and chest wall infections $(26,27)$. Currently, 1,700 robotassisted mitral valve procedures are performed each year (Figure 3). Among 8,814 MV repair procedures reported to the Society of Thoracic Surgeon Database in 2013, 1,132 were performed using robotic assistance, demonstrating a large uptake of the Da Vinci system in the United States.

Robot assisted coronary surgery on the other hand, was pioneered and standardized by several centers in Europe, with surgical activity relatively constant over the past 15 years (Figure 2). At present, approximately 100 procedures per year are performed in Europe, including robot-enhanced MIDCAB or TECAB for single or multiple vessel revascularization. The current literature shows mortality ranging between $0-1 \%$, as well as low rates of post-operative complications, such as revision for bleeding, conversion to a larger incision, and stroke. Another measure of success, vessel patency, has also been maintained in over $90 \%$ of cases $(28,29)$. Furthermore, a decline in conversion rates over the years has been demonstrated (16). As a result, the Da Vinci technology has been adopted by several centres in the United States, with a progressive increase in case numbers (in 2011, over a thousand operations were performed) (Figure 4). Despite surgeon-, team- and institution-specific learning curves, robot-assisted coronary 
Robot-assisted CABG

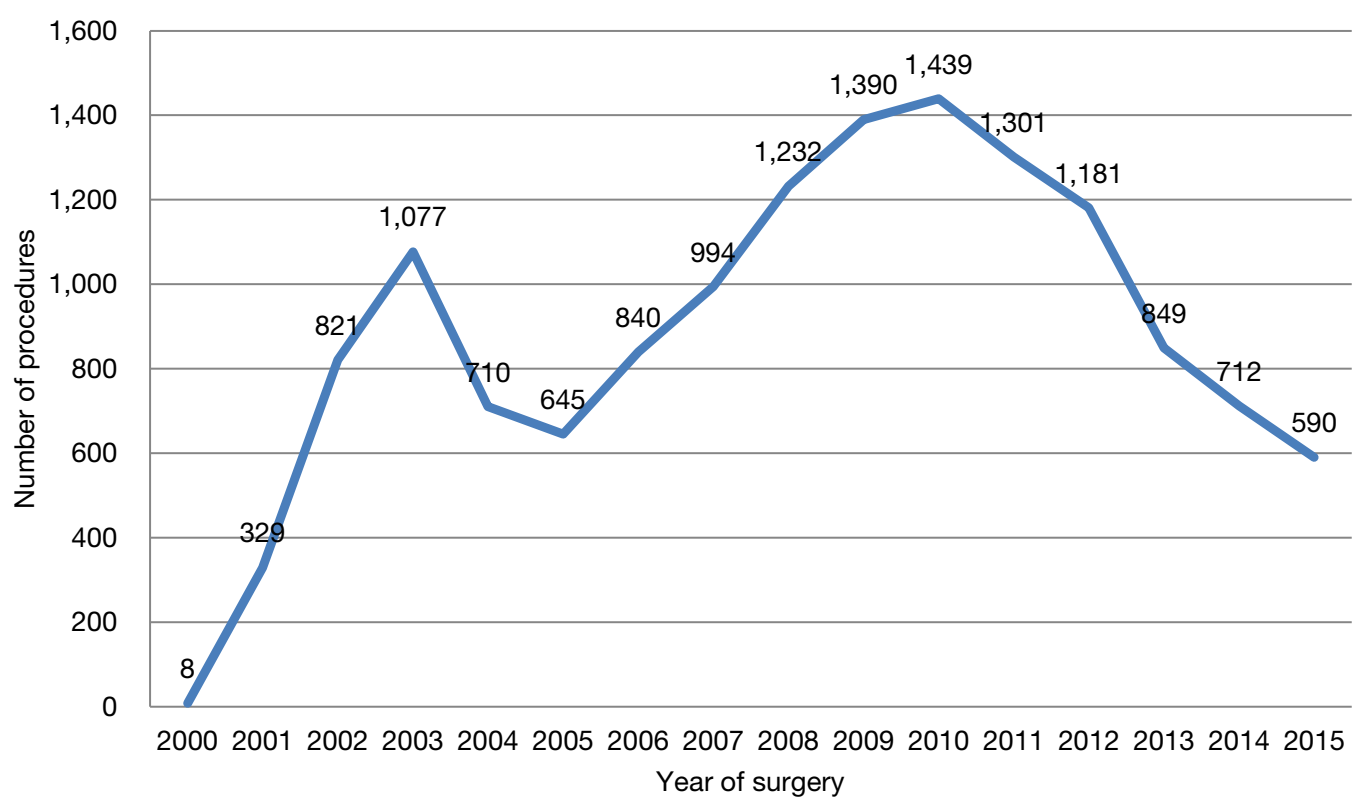

Figure 4 Robot-assisted CABG in the United States. CABG, coronary artery bypass grafting.

surgery has continued to demonstrate favourable outcomes.

Looking forward, the third generation of robotic devices is currently available, and allows complex multi-vessel procedures to be performed routinely on both the arrested and beating heart.

According to current data, robot-enhanced MV repair and TECAB appears safe and effective with the advantage of less invasive surgery. Learning curves are unavoidable with the development of new technologies, however, and must be considered as new programs are initiated. Whilst robotic cardiac surgery has evolved and its use has spread since its initial introduction, these procedures remain in their primitive stages. It is likely that, for the foreseeable future, robotic cardiac surgery will remain in the hands of committed teams at specialized centers (28).

\section{Acknowledgements}

We thank Intuitive Surgical Inc. for providing valuable data to conduct this research.

\section{Footnote}

Conflicts of Interest: The authors have no conflicts of interest to declare.

\section{References}

1. Carpentier A, Loulmet D, Aupècle B, et al. Computer assisted open heart surgery. First case operated on with success. C R Acad Sci III 1998;321:437-42.

2. Falk V, Autschbach R, Krakor R, et al. Computerenhanced mitral valve surgery: toward a total endoscopic procedure. Semin Thorac Cardiovasc Surg 1999;11:244-9.

3. Lehr EJ, Rodriguez E, Chitwood WR. Robotic cardiac surgery. Curr Opin Anaesthesiol 2011;24:77-85.

4. Carpentier A, Loulmet D, Aupecle B, et al. Computerassisted cardiac surgery. Lancet 1999;353:379-80.

5. Autschbach R, Onnasch JF, Falk V, et al. The Leipzig experience with robotic valve surgery. J Card Surg 2000;15:82-7.

6. Onnasch JF, Schneider F, Falk V, et al. Five years of less invasive mitral valve surgery: from experimental to routine approach. Heart Surg Forum 2002;5:132-5.

7. Folliguet T, Vanhuyse F, Constantino X, et al. Mitral valve repair robotic versus sternotomy. Eur J Cardiothorac Surg 2006;29:362-6.

8. Deeba S, Aggarwal R, Sains P, et al. Cardiac robotics: a review and St. Mary's experience. Int J Med Robot 2006;2:16-20.

9. Musumeci F, Mariscalco G, Ranocchi F, et al. Transareolar Robotic-Assisted Access to the Mitral Valve. Innovations 
(Phila) 2015;10:438-40.

10. Loulmet D, Carpentier A, d'Attellis N, et al. Endoscopic coronary artery bypass grafting with the aid of robotic assisted instruments. J Thorac Cardiovasc Surg 1999;118:4-10.

11. Cichon R, Kappert U, Schneider J, et al. Roboticenhanced arterial revascularization for multivessel coronary artery disease. Ann Thorac Surg 2000;70:1060-2.

12. Mohr FW, Falk V, Diegeler A, et al. Computer-enhanced "robotic" cardiac surgery: experience in 148 patients. J Thorac Cardiovasc Surg 2001;121:842-53.

13. Kappert U, Schneider J, Cichon R, et al. Development of robotic enhanced endoscopic surgery for the treatment of coronary artery disease. Circulation 2001;104:I102-7.

14. Bonatti J, Schachner T, Bonaros N, et al. Ongoing procedure development in robotically assisted totally endoscopic coronary artery bypass grafting (TECAB). Heart Surg Forum 2005;8:E287-91.

15. de Cannière D, Wimmer-Greinecker G, Cichon R, et al. Feasibility, safety, and efficacy of totally endoscopic coronary artery bypass grafting: multicenter European experience. J Thorac Cardiovasc Surg 2007;134:710-6.

16. Schachner T, Bonaros N, Wiedemann D, et al. Predictors, causes, and consequences of conversions in robotically enhanced totally endoscopic coronary artery bypass graft surgery. Ann Thorac Surg 2011;91:647-53.

17. Mohr FW, Falk V, Diegeler A, et al. Minimally invasive port-access mitral valve surgery. J Thorac Cardiovasc Surg 1998;115:567-74; discussion 574-6.

18. Vanermen H, Wellens F, De Geest R, et al. Videoassisted Port-Access mitral valve surgery: from debut to routine surgery. Will Trocar-Port-Access cardiac surgery ultimately lead to robotic cardiac surgery? Semin Thorac Cardiovasc Surg 1999;11:223-34.

19. Torracca L, Lapenna E, De Bonis M, et al. Minimally invasive mitral valve repair as a routine approach in

Cite this article as: Pettinari $M$, Navarra E, Noirhomme P, Gutermann H. The state of robotic cardiac surgery in Europe. Ann Cardiothorac Surg 2017;6(1):1-8. doi: 10.21037/ acs.2017.01.02 selected patients. J Cardiovasc Med (Hagerstown) 2006;7:57-60.

20. Greco E, Barriuso C, Castro MA, et al. Port-Access cardiac surgery: from a learning process to the standard. Heart Surg Forum 2002;5:145-9.

21. Casselman FP, Van Slycke S, Wellens F, et al. Mitral valve surgery can now routinely be performed endoscopically. Circulation 2003;108 Suppl 1:II48-54.

22. McClure RS, Athanasopoulos LV, McGurk S, et al. One thousand minimally invasive mitral valve operations: early outcomes, late outcomes, and echocardiographic followup. J Thorac Cardiovasc Surg 2013;145:1199-206.

23. Robicsek F. Robotic cardiac surgery: time told! J Thorac Cardiovasc Surg 2008;135:243-6.

24. Morgan JA, Thornton BA, Peacock JC, et al. Does robotic technology make minimally invasive cardiac surgery too expensive? A hospital cost analysis of robotic and conventional techniques. J Card Surg 2005;20:246-51.

25. Rodriguez E, Nifong LW, Chu MW, et al. Robotic mitral valve repair for anterior leaflet and bileaflet prolapse. Ann Thorac Surg 2008;85:438-44; discussion 444.

26. Suri RM, Taggarse A, Burkhart HM, et al. Robotic Mitral Valve Repair for Simple and Complex Degenerative Disease: Midterm Clinical and Echocardiographic Quality Outcomes. Circulation 2015;132:1961-8.

27. Murphy DA, Moss E, Binongo J, et al. The Expanding Role of Endoscopic Robotics in Mitral Valve Surgery: 1,257 Consecutive Procedures. Ann Thorac Surg 2015;100:1675-81; discussion 1681-2.

28. Lee JD, Srivastava M, Bonatti J. History and current status of robotic totally endoscopic coronary artery bypass. Circ J 2012;76:2058-65.

29. Schachner T, Feuchtner GM, Bonatti J, et al. Evaluation of robotic coronary surgery with intraoperative graft angiography and postoperative multislice computed tomography. Ann Thorac Surg 2007;83:1361-7. 